\title{
Perspective
}

\section{New Opportunities for Breastfeeding Promotion and Support in WIC: Review of WIC Food Packages, Improving Balance and Choice}

Kathleen M. Rasmussen, ScD, RD ${ }^{1}$; Shannon E. Whaley, $\mathrm{PhD}^{2}$; Rafael Pérez-Escamilla, $\mathrm{PhD}^{3}$; A. Catharine Ross, $\mathrm{PhD}^{4}$; Susan S. Baker, MD, $\mathrm{PhD}^{5}$; Tamera Hatfield, MD, $\mathrm{PhD}^{6}$; Marie E. Latulippe, MS, $\mathrm{RD}^{7}$

\begin{abstract}
Although breastfeeding $(\mathrm{BF})$ is the recommended way to feed infants, this may be difficult for the low-income women served by the Special Supplemental Nutrition Program for Women, Infants, and Children (WIC). The 2017 recommended revisions to the WIC food packages provide substantial support to both exclusively and partially BF dyads, remove barriers to partial BF choices within the first 30 days postpartum, and increase flexibility in determining the amount of formula offered to partially breastfed infants. When combined with adequate support and tailored counseling, these changes are intended to make it easier for women served by WIC to choose to breastfeed.
\end{abstract}

Key Words: breastfeeding, WIC, infant formula, food packages (J Nutr Educ Behav. 2017;49:S197-S201.)

Accepted April 20, 2017.

\section{INTRODUCTION}

Health authorities around the world and in the US ${ }^{2}$ recommend that infants be breastfed exclusively for about the first 6 months of life followed by the introduction of complementary foods and continued BF until at least age 1 . These recommendations are based on literature that documented that breastfeeding (BF) provides benefits to the health of both BF women and their breastfed infants. ${ }^{3}$ In the US, these recommendations are the basis for public health goals related to $\mathrm{BF} .{ }^{4}$ The Healthy People 2020 goal is that $89.1 \%$ of women should initiate BF; currently $81.1 \%$ women in the US are meeting this goal. ${ }^{5}$ However, US women have been less successful in meeting goals for the duration of either partial or exclusive $\mathrm{BF}$ at 6 months. In fact, only $51.8 \%$ of women are partially BF at 6 months and only $22.3 \%$ are doing so exclusively, compared with national goals of $60.6 \%$ and $25.5 \%$, respectively. ${ }^{4,5}$

Breastfeeding is a socially patterned behavior that varies among racial and

\footnotetext{
${ }^{1}$ Division of Nutritional Sciences, Cornell University, Ithaca, NY

${ }^{2}$ Division of Research and Evaluation, Public Health Foundation Enterprises WIC Program, Los Angeles, CA

${ }^{3}$ Department of Chronic Disease Epidemiology, Yale School of Public Health, New Haven, CT ${ }^{4}$ Department of Nutritional Sciences, Pennsylvania State University, University Park, PA

${ }^{5}$ Women and Children's Hospital of Buffalo, Buffalo, NY

${ }^{6}$ Division of Maternal Fetal Medicine, Department of Obstetrics and Gynecology, University of California Irvine Medical Center, Orange, CA

${ }^{7}$ Health and Medicine Division, Food and Nutrition Board, National Academies of Sciences, Engineering, and Medicine, Washington, DC

Conflict of Interest Disclosure: The authors' conflict of interest disclosures can be found online with this article on www.jneb.org.

Address for correspondence: Kathleen M. Rasmussen, ScD, RD, 111 Savage Hall, Division of Nutritional Sciences, Cornell University, Ithaca, NY 14853-6301; Phone: (607) 255-2290; Fax: (607) 255-1033; E-mail: kathleen.rasmussen@cornell.edu

(C2017 Society for Nutrition Education and Behavior. Published by Elsevier, Inc. All rights reserved.
}

http://dx.doi.org/10.1016/j.jneb.2017.04.007 ethnic groups. It is highest among Hispanic women, although with strong variation across subgroups of different national origins, and is lowest among non-Hispanic black women. ${ }^{6}$ In addition, women with lower incomes ${ }^{6}$ or less education, or who work a job with inadequate support for BF are less likely to choose to breastfeed or to continue to breastfeed for the recommended duration than are women who do not have these characteristics, such as women served by the Special Supplemental Nutrition Program for Women, Infants, and Children (WIC). ${ }^{7,8}$ Although the proportion of women who choose to breastfeed their infants for $\geq 6$ months has been rising both nationally and among women participating in WIC, the proportion of women participating in WIC who breastfeed for this duration has consistently lagged that of all women. ${ }^{9,10}$ Therefore it is crucial to improve BF outcomes among women served by WIC so as to improve their health and provide their infants with the many benefits of being breastfed, and to achieve national public health goals. Doing so is expected to reduce national health care costs. ${ }^{8}$

Improving the adoption and continuation of BF is central to the mission of WIC. This was reaffirmed in the latest policy analysis and guidance issued by the US Department of Agriculture 
Federal Regulations that Provide Guidance for Implementing WIC's Breastfeeding Support ${ }^{10}$

- WIC is required to make breastfeeding promotion and support available to all participants

(7 CFR 246.11)

- " $[A]$ II staff have a responsibility to encourage, educate, and support women in their breastfeeding decisions and have a basic knowledge of breastfeeding and understand their unique role in order to effectively support breastfeeding as the standard method of infant feeding" (7 CFR 246.3[3])

- State agencies are required "to provide training on the promotion and management of breastfeeding to local agency staff who will provide information and assistance on breastfeeding to participants" (7 CFR 246.11[c][2])

- State agencies are required to "develop policies that create a positive breastfeeding environment that endorses breastfeeding as the standard method of infant feeding" (7 CFR $246.11[c][7][i])$

- Local agencies are required to "have a plan to ensure that women have access to breastfeeding promotion and support activities during the prenatal and postpartum periods" (7 CFR 246.11[d])

Figure. Federal regulations that provide guidance for implementing Special Supplemental Nutrition Program for Women, Infants, and Children's (WIC's) breastfeeding support. ${ }^{10}$ CFR indicates Code of Federal Regulations.

(USDA). ${ }^{11}$ Moreover, WIC's support of $\mathrm{BF}$ is subject to specific federal regulations (Figure). The USDA has a national BF promotion and support campaign known as Loving Support Makes Breastfeeding Work, which provides the foundation for BF education as well as counseling and support efforts in WIC. ${ }^{12}$ Unfortunately, BF support and promotion activities are not uniformly accessible in all states, which may reflect differences in available resources or the needs of the populations served. ${ }^{13}$

To help WIC meet its stated goals to support BF, the 2006 recommendations for revisions ${ }^{14}$ suggested substantial changes to the food packages for women and their infants. Included in the 2006 recommendations was an enhancement of the food packages for exclusively BF dyads as well as additional foods for breastfed infants from 6 to 12 months. ${ }^{4}$ Nearly all of these recommendations were implemented in 2009. The current food package for breastfed infants aged 0-1 month con- tains no infant formula. The 2006 committee based this on literature showing that delayed introduction of formula to breastfed infants was associated with longer BF than early introduction of formula. ${ }^{14}$ In the first 30 days postpartum, states currently have the option to issue 1 can of powdered infant formula to BF women (that reconstitutes to $104 \mathrm{fl} \mathrm{oz}$ ) on a case-by-case basis, but implementation of this option varies by state. Women who are BF and need infant formula within the first 30 days (or $>1$ can in states that allow the 1-can option) may be issued the fully formula-fed package for the infant (which contains $823 \mathrm{fl}$ oz in 9 cans) but cannot be issued the fully BF food package for themselves. ${ }^{15}$

Results from a large study conducted in 17 WIC agencies suggested that when this recommendation was implemented in 2009, it created an apparent forced choice between exclusive BF and exclusive formula feeding. The proportion of women who chose exclusive BF rose slightly, as intended. However, the proportion of women enrolled in WIC who chose exclusive formula feeding rose much more, which was not intended. There was a corresponding decrease in partial BF. ${ }^{16}$ The exception to this multi-state trend occurred in California, probably as a result of investments made to prepare WIC staff and participants for the implementation of the revised WIC food packages. ${ }^{17}$ The results in California provided evidence that the forced choice could be prevented by assessing the motherchild dyad early, often during the prenatal period, and by supporting the transition to exclusive or partial BF instead of formula feeding. However, for the great majority of the country, the 2009 policy changes were not associated with an increase in the proportion of WIC participants who chose to breastfeed.

Approximately $69 \%$ of the 1.94 million infants served by WIC in 2015-2016 received infant formula. ${ }^{18}$ This formula has a high market value. Although the amount of infant formula in the food packages for infants was reduced when the 2006 recommendations for revisions to the food packages were implemented by the USDA in 2009 , the market value (and thus perceived value) of the infant formula provided by WIC varies with infant age and currently ranges from $\$ 155.28$ to $\$ 177.20$ per month for the fully formula-fed infant. ${ }^{13}$ The market value of the packages for partially breastfed infants also varies by age: $\$ 79.39$ for ages $1-3$ months, \$95.49 for ages 4-5 months, and \$96.86 for ages 6-11 months. In contrast, the market value of packages for breastfed infants ranges from $\$ 0$ (for the fully breastfed infant from 0 to 5 months) to $\$ 79.68$ per month (for the fully breastfed infant from 6 to 11 months). In addition to the differences in value for infant packages, there are also differences in the market value of food packages for mothers based on their choice of infant-feeding method. For 6 months, mothers of fully formulafed infants receive a food package with a current market value of $\$ 46.58 / \mathrm{mo}$. In contrast, mothers of partially and fully BF infants receive food packages with a current market values of $\$ 58.70$ and $\$ 74.16$, respectively, and these food packages can be received for up to 12 months to support their additional caloric needs. ${ }^{13}$ This difference 
in the annual market value of the food packages is substantial: For exclusively or partially breastfed infants and their mothers, this value is estimated to be $\$ 1,368$ and $\$ 1,734$, respectively; for formula-fed infants and their mothers, this value is estimated to be $\$ 2,211$. $^{13}$ This difference in value may influence women's choice regarding how to feed their infants, ${ }^{13,19}$ and thus conflicts with WIC's stated support for BF.

\section{DISCUSSION}

To address these several important challenges related to promotion, support, and protection of BF within WIC, the Committee to Review WIC Food Packages (the committee), identified several critical areas to support BF in its 2017 report. ${ }^{13}$ First, when developing the food packages, the committee decided that it was essential to consider the dyadic nature of $\mathrm{BF}$ (eg, when the infant is breastfed, the mother has greater nutritional needs related to milk production). Second, given the social, cultural, and other barriers to BF faced by WIC participants, the committee recognized that currently WIC may be reaching nearly all of those participants who are willing and able to breastfeed exclusively. However, many women remain who are interested in BF and want to try it but, for various reasons beyond WIC's control, are unable to breastfeed exclusively. As a result, it is important to address the needs of women who choose partial BF in terms of counseling support and an appropriate food package. Third, the committee continued to recognize the first 30 days postpartum as a highly sensitive period when women need qualified support to achieve successful BF, whether exclusive or partial. The committee fully endorsed current WIC policy to support $\mathrm{BF}^{11}$ and further enhanced the vision for support of BF in the first 30 days by explicitly stating that "all women should receive adequate counseling and support for BF prenatally through the first month postpartum." ${ }^{13}$

To recognize the dyadic nature of $\mathrm{BF}$, to promote and support it among participating women, and to address the higher nutritional needs of BF compared with formula-feeding women, the committee recommended changes to the food packages. These changes included a higher monthly cash value voucher, which can be used to purchase fresh or frozen vegetables and fruits, for women who breastfeed exclusively or partially (\$35 or $\$ 25$, respectively) than for women who are not BF (\$15). The proposed changes included additional canned fish every 3 months for women who breastfeed exclusively or partially (60 or $30 \mathrm{oz}$, respectively) than for women who are not $\mathrm{BF}(10 \mathrm{oz})$. As a result of these and other changes to the food packages, the monthly market value of the food packages would increase by $\$ 4.55$ and $\$ 5.43$ for women who breastfeed exclusively or partially, respectively, compared with $\$ 0.56$ for food packages for women who feed only infant formula. ${ }^{13}$ For women who are interested in $\mathrm{BF}$, this increase in the market value of their food packages that would start immediately postpartum was intended to create up-front incentives for BF that support their infant-feeding decisions. Finally, the revised packages would permit issuance of a partial BF package in the first 30 days postpartum, which is not currently allowed. The intention of this recommendation was to support all levels of BF so that more mothers could achieve their BF goals. To reduce the possibility that this recommendation would interfere with the establishment of participants' desired intensity and duration of $\mathrm{BF}$, the committee recommended issuance of infant formula in up-to amounts across all packages. This approach would allow WIC staff to tailor issuance of infant formula to the amount needed. This was intended to enhance the discussion of infant feeding with every mother of a newborn so that the nutrition assessment and counseling provided by WIC staff would support the needs of each mother-infant dyad. Up-to amounts would also support partial BF by using the minimal amount of formula that mothers needed to continue to breastfeed.

If implemented nationally, this approach would provide a standard of care to support BF as well as incentives for $\mathrm{BF}$ in a larger proportion of the WIC population than is currently the case. Setting a standard for intensive support in the early postpartum period is likely to attract more interest in exclusive $\mathrm{BF}$ as well as enable others to attempt BF secure in the knowledge that partial BF may be an acceptable alternative for those with barriers to exclusivity. Moreover, because partial
BF can occur over a much longer period than exclusive $\mathrm{BF}$, preventing a forced choice may allow many more infants enrolled in WIC to receive the benefits of $\mathrm{BF}$ for a longer period than at present. In fact, the committee anticipated that $5 \%$ of women who currently feed formula would switch to partial BF in response to the incentives in the revised food packages. ${ }^{13}$ Additional mechanisms for supporting these choices may indeed increase the number of partially $\mathrm{BF}$ women beyond these projections. If the projected $5 \%$ change occurred, the committee's regulatory impact analysis suggested that this would create a modest cost savings. ${ }^{13}$ This would occur because the savings from not issuing the full amount of infant formula to partially breastfed infants would exceed the increased cost of issuing more substantial food packages to women who partially breastfeed than to women who feed only infant formula.

The committee recognized that enhancing BF would require expansion and coordination of WIC resources that promote and support $\mathrm{BF}$ as well as consistent funding for these activities. Most funding for BF support comes from Nutrition Services Administration funds, which are included in the general WIC funding allocation. Additional funding for Breastfeeding Peer Counseling is determined on an annual basis and support from peer counseling support is not available in all states for all WIC participants. The committee encouraged the USDA Food and Nutrition Service (FNS) to meet this funding challenge so that staff could provide support to all participants who wished to breastfeed. ${ }^{13}$

Lessons from California provide robust evidence that BF support is important to successful implementation of policy changes. When BF support and promotion efforts were paired with the 2009 changes in the food packages in California, the proportion of women who chose to fully breastfeed increased. ${ }^{17}$ Moreover, the increases in full formula feeding observed in other states did not occur in California. ${ }^{16,17}$ The specific activities implemented started 9 months before the food package changes and included 2 statewide education campaigns: 1 focused on staff wellness, including education about the new foods and upcoming changes to the BF packages. The other started 6 months before 
the changes and focused on pregnant, postpartum, and BF women. The proportion of women who chose the fully BF packages started to increase before the actual food package changes, and then increased further after the changes. This suggests that policy changes are maximally effective when paired with BF support and education.

An example from a smaller area came from the Erie County WIC program in New York, where WIC staff members visited every local maternity hospital every other day. This permitted staff to identify women who participated in WIC prenatally as well as women who could be recruited postnatally, providing them with early support for BF and timely support for problems that might develop. Together, these examples suggested possibilities that may be effective in both small and large WIC agencies.

To fulfill its mission to support all women and ensure that all infants have adequate nutrition regardless of the mother's chosen method of infant feeding, WIC also provides formula for the infants of mothers who do not wish to breastfeed or cannot. The 2006 recommendations (implemented by the USDA in 2009) calibrated formula amounts to meet nearly $100 \%$ of the caloric needs of fully formula-fed infants and increased amounts at 4-5 months as caloric needs increased. ${ }^{4}$ The committee did not recommend further reductions in the amount of formula provided to fully formula-fed infants. It was concerned that reducing the amount of infant formula would impose a financial burden on families served by the program or lead to the over-dilution of formula when no suitable, affordable alternatives were available for them. ${ }^{20}$ Moreover, the committee concluded that until additional resources are made available to USDA-FNS to meet the demand for BF support services, it would continue to be difficult for many WIC participants to meet their prenatal BF goals. Finally, the committee recommended that the provision of formula by WIC should be reconsidered at the time of the next review, ${ }^{13}$ because the global evidence indicated that this is a major disincentive for BF, especially among low-income women, and is inconsistent with the World Health Organization Code for Marketing of Breast Milk Substitutes. $^{21,22}$

\section{IMPLICATIONS FOR RESEARCH AND PRACTICE}

Inasmuch as BF is supported by multiple sources of funding that must be ongoing and coordinated for maximal impact, revisions to the food packages are only part of what must be in place for WIC to achieve its BF objectives. The committee recommended changes to the food packages that provide both immediate (an improved food package) and extended (if the mother continues to breastfeed) nutritional support for BF woman. The committee recommended that the USDA-FNS

\section{fund data collection and analysis of that data toward optimizing the support for breastfeeding and increasing the proportion of WIC participants who choose to initiate and continue breastfeeding, and tailoring the food package to best meet the needs and goals of the breastfeeding dyad. ${ }^{13}$}

The committee encouraged the USDA-FNS to meet the challenge of coordinating the resources necessary to achieve WIC's BF objectives.

The time of transition from 1 food package to another is important for research because it permits a before vs after comparison of implementation of package changes, which can be studied in several locales simultaneously and with variation in the method of implementation. The committee noted that "it would be ideal to test different approaches for the promotion of BF through randomized trials" ${ }^{\prime 13}$ with adequate representation of the various WIC subpopulations. Regardless of the method used to study BF outcome, the committee recommended that the USDA-FNS develop "a set of universal breastfeeding indicators that can be captured across [WIC Management Information Systems]."13 In the long term, however, it will be necessary for the USDAFNS to study the impact and costeffectiveness of the food packages separately and in conjunction with the program services as motivators of the choices to both initiate and continue exclusive and partial BF for the recommended durations. Lessons learned from such research could inform the development of even more effective ways to protect, promote, and support BF within WIC.

To support BF, WIC relies on WIC paraprofessional staff as well as BF peer counselors and staff members who are Certified Lactation Consultants and International Board Certified Lactation Consultants. It was outside the committee's task to make recommendations on this subject, but if the committee's proposed changes to the food packages are implemented and successful in motivating women to try and continue to breastfeed, full coverage by these different types of counselors and consultants to meet the demand is likely necessary. ${ }^{23}$ This would be an appropriate time for WIC programs and the USDA-FNS to identify the level of investment needed to provide full support nationally for initiation and continuation of BF.

The Special Supplemental Nutrition Program for Women, Infants, and Children has a pivotal role in achieving national and WIC-specific goals for BF. It is difficult to increase the proportion of women who participate in WIC to initiate and continue BF because of the many societal barriers to BF experienced by these women, as well as other lowincome women. It may also be difficult to increase the proportion of women who participate in WIC and who breastfeed because of the greater financial incentive provided to women enrolled in WIC for formula-feeding compared with BF. For many women who participate in WIC, infant formula is essential because $\mathrm{BF}$ is not desired or feasible. For many other women enrolled in WIC, the opposite is true: they want to breastfeed and need help to make it feasible. For these women, the committee offered a wide range of incentives to breastfeed and fewer disincentives, and a call for strengthening and coordinating the WIC resources for $\mathrm{BF} .^{13}$

\section{ACKNOWLEDGMENTS}

This work was derived from the National Academies of Sciences, Engineering, and Medicine (The National Academies) report "Review of WIC food packages: improving balance and choice." The authors are responsible for the content of this article, which does not represent the views of The National Academies. 


\section{REFERENCES}

1. World Health Organization. Infant and young child feeding. http://who.int/ mediacentre/factsheets/fs342/en/. Accessed January 6, 2017.

2. Kleinman RE, Greer RF, eds. Pediatric Nutrition. 7th ed. Elk Grove Village, IL: American Academy of Pediatrics; 2014.

3. Review of WIC food packages: proposed framework for revisions: interim report. National Academies of Sciences, Engineering, and Medicine. https://www.nap.edu/ catablog/21832/review-of-wic-food-packag es-proposed-framework-for-revisions-inte rim. Accessed February 28, 2017.

4. Review of WIC food packages: improving balance and choice: final report. National Academies of Sciences, Engineering, and Medicine. The National Academies. https://www.nap.edu/catalog/ 23655/review-of-wic-food-packagesimproving-balance-and-choice-final. Accessed January 5, 2017.

5. Healthy People 2020: Maternal, infant, and child health objectives. US Department of Health and Human Services. https://www.healthypeople.gov/2020/ topics-objectives/topic/maternal-infantand-child-health/objectives. Accessed January 6, 2017.

6. Breastfeeding Report Card-United States, 2016. https://www.cdc.gov/breast feeding/pdf/2016breastfeedingreportcard. pdf. Accessed January 6, 2017.

7. National Immunization Survey (NIS), 2016. https://www.cdc.gov/breastfeeding/ data/nis_data/index.htm. Accessed January 6, 2017.
8. Pérez-Escamilla R, Sellen D. Equity in breastfeeding: where do we go from here? J Hum Lactation. 2015;31:12-14.

9. Bartick MC, Jegier BJ, Green BD, Schwarz EB, Reinhold AG, Stuebe AM. Disparities in breastfeeding: impact on maternal and child health outcomes and costs. J Pediatr. 2017 Feb;181:49-55.e6.

10. Nutrition, physical activity and obesity data, trends and maps web site. Centers for Disease Control and Prevention. https://nccd.cdc.gov/NPAO_DTM/. Accessed January 6, 2017.

11. Ryan A, Wenjun Z, Acosta A. Breastfeeding continues to increase into the new millennium. Pediatrics. 2002;11:1103-1109.

12. Breastfeeding policy and guidance. USDA, FNS. http://www.fns.usda.gov/ sites/default/files/wic/WIC-BreastfeedingPolicy-and-Guidance.pdf. Accessed January 6, 2017.

13. USDA. Loving support makes breastfeeding work. https://lovingsupport.fns. usda.gov/. Accessed January 6, 2017.

14. Institute of Medicine. WIC food packages: time for a change. https://www. nap.edu/catalog/11280/wic-food-packagestime-for-a-change. Accessed January 6, 2017.

15. Special Supplemental Nutrition Program for Women, Infants, and Children (WIC). Revisions in the WIC food packages. Interim Rule. USDA, FNS. 7 C.F.R. \$246. December 6, 2007.

16. Special Supplemental Nutrition Program for Women, Infants, and Children (WIC). Revisions in the WIC food packages. Final rule. USDA, FNS. 7 C.F.R. S 246. March 4, 2014.
17. Wilde P, Wolf A, Fernandez M, Collins A. Evaluation of the birth month breastfeeding changes to the WIC food packages. http://www.fns.usda.gov/sites/default/ files/BirthMonth.pdf. Accessed January 6, 2017.

18. Whaley SE, Koleilat M, Whaley M, Gomez J, Meehan K, Saluja K. Impact of policy changes on infant feeding decisions among low-income women participating in the Special Supplemental Nutrition Program for Women, Infants, and Children. Am J Pub Health. 2012; 102:2269-2273.

19. USDA, FNS. WIC program. http:// www.fns.usda.gov/pd/wic-program. Accessed January 6, 2017.

20. Jensen E, Labbok M. Unintended consequences of the WIC formula rebate program on infant feeding outcomes: will the new food packages be enough? Breastfeed Med. 2011;6:145-149.

21. Burkhardt MC, Beck AF, Kahn RS, Klein MD. Are our babies hungry? Food insecurity among infants in urban clinics. Clin Pediatr. 2012;51: 238-243.

22. Pérez-Escamilla R, Curry L, Minhas D, Taylor L, Bradley E. Scaling up of breastfeeding promotion programs in low- and middle-income countries: the "breastfeeding gear" model. Adv Nutr. 2012;3:790-800.

23. Pérez-Escamilla R, Martinez JL, Segura-Pérez S. Impact of the Babyfriendly Hospital Initiative on breastfeeding and child health outcomes: a systematic review. Matern Child Nutr. 2016;12:402-417. 


\section{CONFLICT OF INTEREST}

The authors have not stated any conflicts of interest. 